\title{
LINEAR INTERVAL INEQUALITIES
}

\author{
JiŘí Rohn, JAna KRESLOVÁ \\ Faculty of Mathematics and Physics, Charles University \\ Malostranské nám. 25, 11800 Prague, Czech Republic
}

\section{6 .1993}

\begin{abstract}
We prove that a system of linear inequalities with interval-valued data is weakly solvable (each system obtained by fixing coefficients in the intervals prescribed has a solution) if and only if it is strongly solvable (all such systems have a solution in common) and describe an algorithm for checking strong solvability.
\end{abstract}

\section{Introduction}

In this paper we study systems of linear interval inequalities

$$
A^{I} x \leq b^{I}
$$

where $A^{I}=\{A ; \underline{A} \leq A \leq \bar{A}\}$ (componentwise inequalities) is an $m \times n$ interval matrix and $b^{I}=\{b ; \underline{b} \leq b \leq \bar{b}\}$ is an $m$-dimensional interval vector. Under the formally written system (1) we understand the family of systems of linear inequalities

$$
A x \leq b
$$

for all $A$ and $b$ satisfying

$$
A \in A^{I}, \quad b \in b^{I} .
$$

We introduce two concepts of solvability: 
1. a system (1) is called weakly solvable if for each $A \in A^{I}, b \in b^{I}$ the system (2) has a solution (which generally depends on $A$ and $b$ )

2. a system (1) is called strongly solvable if there exists an $x_{0}$ satisfying $A x_{0} \leq b$ for each $A \in A^{I}, b \in b^{I}$ (i.e., if all the systems (2), (3) have a solution in common).

We prove in Theorem 1 below that, rather surprisingly, weak and strong solvability are equivalent, and describe a method for verifying strong (equivalently, weak) solvability of (1) by means of solving only one system of linear (not interval) inequalities. As a by-product of the proof, we obtain that if (1) is not strongly solvable, then it contains an unsolvable system $A_{0} x \leq \underline{b}$ with a matrix $A_{0} \in A^{I}$ of a very special form: for each $j$, all but at most one of the coefficients of the $j$-th column of $A_{0}$ are fixed either at the lower, or at the upper bound of the respective interval (Theorem 2), and we describe a method for constructing such a matrix $A_{0}$ via solving an auxiliary linear programming problem. The results obtained are summed up in the form of an algorithm. Several relevant results are mentioned in the concluding remarks.

\section{The results}

To facilitate formulations, let us call a vector $x_{0}$ a strong solution of (1) if

$$
A x_{0} \leq b
$$

holds for each $A \in A^{I}, b \in b^{I}$; hence, the above-defined strong solvability is equivalent to the existence of a strong solution to (1). Let us denote the set of all strong solutions of (1) by $X_{S}$. We have this description:

Proposition. For a system (1) we have

$$
X_{S}=\left\{x_{1}-x_{2} ; \bar{A} x_{1}-\underline{A} x_{2} \leq \underline{b}, x_{1} \geq 0, x_{2} \geq 0\right\} .
$$

Proof. Let $x \in X_{S}$. Put $x_{1}=x^{+}=\max \{x, 0\}$ (componentwise) and $x_{2}=x^{-}=\max \{-x, 0\}$. Then $x_{1} \geq 0, x_{2} \geq 0$ and $x=x_{1}-x_{2}$. Furthermore define a matrix $A$ columnwise by $A_{. j}=\bar{A}_{. j}$ if $x_{j} \geq 0$ and $A_{. j}=\underline{A}_{. j}$ if $x_{j}<0$, 
so that $A \in A^{I}$. Then, since $x$ is a strong solution, we have $\bar{A} x_{1}-\underline{A} x_{2}=$ $A x \leq \underline{b}$. Conversely, let $x_{1}, x_{2}$ be a nonnegative solution to $\bar{A} x_{1}-\underline{A} x_{2} \leq \underline{b}$ and let $x=x_{1}-x_{2}$. Then for each $A \in A^{I}, b \in b^{I}$ we have that $A x=$ $A\left(x_{1}-x_{2}\right) \leq \bar{A} x_{1}-\underline{A} x_{2} \leq \underline{b} \leq b$, hence $x$ is a strong solution.

Now we have this main result:

Theorem 1 A system of linear interval inequalities (1) is weakly solvable if and only if it is strongly solvable.

We shall prove Theorem 1 together with this result:

Theorem 2 Let a system (1) be not strongly solvable. Then it contains an unsolvable system

$$
A_{0} x \leq \underline{b}
$$

where $A_{0}$ is of the following form: for each $j \in\{1, \ldots, n\}$ there is an $i_{j} \in$ $\{1, \ldots, m\}$ such that

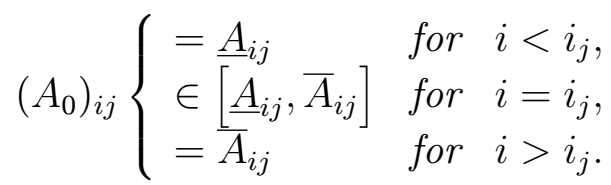

Proof of Theorems 1 and 2. Obviously, a strongly solvable system (1) is also weakly solvable. To prove Theorem 2 and also the "only if" part of Theorem 1, assume that (1) is not strongly solvable. Then, according to the Proposition, the system of linear inequalities

$$
\begin{aligned}
\bar{A} x_{1}-\underline{A} x_{2} & \leq \underline{b} \\
x_{1} \geq 0, \quad x_{2} & \geq 0
\end{aligned}
$$

does not have a solution, which in view of Farkas lemma implies the existence of a vector $y \geq 0$ satisfying $\bar{A}^{T} y \geq 0, \underline{A}^{T} y \leq 0$ and $\underline{b}^{T} y<0$. For each $j \in\{1, \ldots, n\}$ and $k \in\{0, \ldots, m\}$ define a number $t_{k}^{j}$ by

$$
t_{k}^{j}=\sum_{i \leq k} y_{i} \underline{A}_{i j}+\sum_{i>k} y_{i} \bar{A}_{i j}
$$


(employing a usual convention that $\sum_{\emptyset}=0$ ). Then we have

$$
\prod_{k=1}^{m} t_{k-1}^{j} t_{k}^{j}=\left(\prod_{k=1}^{m-1} t_{k}^{j}\right)^{2} t_{0}^{j} t_{m}^{j}=\left(\prod_{k=1}^{m-1} t_{k}^{j}\right)^{2}\left(\bar{A}^{T} y\right)_{j}\left(\underline{A}^{T} y\right)_{j} \leq 0
$$

hence there exists a $k \in\{1, \ldots, m\}$ satisfying

$$
t_{k-1}^{j} t_{k}^{j} \leq 0
$$

But since

$$
t_{k-1}^{j} t_{k}^{j}=\left(\sum_{i<k} y_{i} \underline{A}_{i j}+y_{k} \bar{A}_{k j}+\sum_{i>k} y_{i} \bar{A}_{i j}\right)\left(\sum_{i<k} y_{i} \underline{A}_{i j}+y_{k} \underline{A}_{k j}+\sum_{i>k} y_{i} \bar{A}_{i j}\right),
$$

we see that there exists an $\alpha^{j} \in\left[\underline{A}_{k j}, \bar{A}_{k j}\right]$ such that

$$
\sum_{i<k} y_{i} \underline{A}_{i j}+y_{k} \alpha^{j}+\sum_{i>k} y_{i} \bar{A}_{i j}=0
$$

holds. Now put $i_{j}=k$ and define $A_{0}$ by

$$
\left(A_{0}\right)_{i j}= \begin{cases}\underline{A}_{i j} & \text { if } i<i_{j} \\ \alpha^{j} & \text { if } i=i_{j} \\ \bar{A}_{i j} & \text { if } i>i_{j}\end{cases}
$$

( $i=1, \ldots, m, j=1, \ldots, n)$, then $A_{0}$ is of the form (4), $A_{0} \in A^{I}$ and from (5) we have $A_{0}^{T} y=0$ which together with $y \geq 0, \underline{b}^{T} y<0$ implies (again using Farkas lemma) that the system of linear inequalities

$$
A_{0} x \leq \underline{b}
$$

does not have a solution. This proves Theorem 2, and by contradiction also the "only if" part of Theorem 1.

Let us notice that the proof of Theorem 2 gives a method for constructing a matrix $A_{0}$ provided a nonnegative vector $y$ satisfying $\bar{A}^{T} y \geq 0, \underline{A}^{T} y \leq$ $0, \underline{b}^{T} y<0$ is known. Such a vector can be found by solving the linear programming problem

$$
\min \left\{\underline{b}^{T} y ; \bar{A}^{T} y \geq 0, \underline{A}^{T} y \leq 0,0 \leq y \leq e\right\}
$$


where $e=(1,1, \ldots, 1)^{T}$. In fact, the set of feasible solutions of $(6)$ is nonempty ( $y=0$ is feasible) and bounded, hence (6) has a finite optimum. If (1) is not strongly solvable, then from the proof of Theorem 2 (where we can normalize $y$ to achieve $y \leq e$ ) we know that the optimal value of $(6)$ is negative. Hence any optimal solution $y$ to (6) satisfies $\underline{b}^{T} y<0$ and can be used for construction of a matrix $A_{0}$.

We can sum up our results into the form of a simple algorithm:

Algorithm (for checking solvability of (1)).

1. Solve the system of linear inequalities

$$
\begin{gathered}
\bar{A} x_{1}-\underline{A} x_{2} \leq \underline{b} \\
x_{1} \geq 0, \quad x_{2} \geq 0
\end{gathered}
$$

by any known method (e.g. by phase I of the simplex algorithm).

2. If a solution $x_{1}, x_{2}$ to (7) is found, set $x=x_{1}-x_{2}$ and terminate: (1) is strongly solvable and $x$ is a strong solution to it.

3. If (7) does not have a solution, find an optimal solution $y$ of the linear program (6).

4. Using $y$, construct $A_{0}$ as in the proof of Theorem 2 and terminate: (1) is not weakly (nor strongly) solvable and the system $A_{0} x \leq \underline{b}$ does not have a solution.

\section{Concluding remarks}

We conclude with mentioning several related results.

1. The description of the set of strong solutions $X_{S}$ in the Proposition is similar to that one used in [2] for characterizing the set of "tolerance solutions" of a system of linear interval equations.

2. A result related to the form of the matrix $A_{0}$ in Theorem 2 can be found in [3], Theorem 5.1, assertion (C7), where it is proved that if a 
square interval matrix $A^{I}$ contains a singular matrix at all, then it also contains a singular matrix $A_{0}$ of the form

$$
\left(A_{0}\right)_{i j}\left\{\begin{array}{lll}
\in\left\{\underline{A}_{i j}, \bar{A}_{i j}\right\} & \text { for } & (i, j) \neq(k, m) \\
\in\left[\underline{A}_{i, j}, \bar{A}_{i j}\right] & \text { for } & (i, j)=(k, m)
\end{array}\right.
$$

for some pair of indices $(k, m)$.

3. It follows from the result by Gerlach [1] that a vector $x$ satisfies $A x \leq b$ for some $A \in A^{I}, b \in b^{I}$ if and only if it solves a system

$$
\left(\frac{1}{2}(\underline{A}+\bar{A})-\frac{1}{2}(\bar{A}-\underline{A}) S\right) x \leq \bar{b}
$$

for some signature matrix $S$ (i.e., a diagonal matrix with diagonal entries 1 or -1$)$. Since there are altogether $2^{n}$ signature matrices, it appears that the problem of verifying that no system $A x \leq b$ with data satisfying $A \in A^{I}, b \in b^{I}$ has a solution is much more difficult than that of verifying strong solvability.

\section{References}

[1] W. Gerlach, Zur Lösung linearer Ungleichungssysteme bei Störung der rechten Seite und der Koeffizientenmatrix, Math. Operationsforsch. Stat., Ser. Optimization 12 (1981), No. 1, 41-43

[2] J. Rohn, Inner Solutions of Linear Interval Systems, in: Interval Mathematics 1985 (K. Nickel, Ed.), Lect. Notes Comput. Sci. 212, SpringerVerlag, Berlin 1986, 157-158

[3] J. Rohn, Systems of Linear Interval Equations, Linear Algebra Appl. 126 (1989), 39-78. 